December, 2011

\title{
Off-shell Construction of Superconformal Chern-Simons Theories in Three Dimensions
}

\author{
Masato Arai田 and Shin Sasaki胞 \\ ${ }^{\dagger}$ Institute of Experimental and Applied Physics, \\ Czech Technical University in Prague, \\ Horská 3a/22, 128 00, Prague 2, Czech Republic \\ ${ }^{\sharp}$ Department of Physics \\ Kitasato University \\ Sagamihara, 228-8555, Japan
}

\begin{abstract}
We propose an off-shell construction of three-dimensional $\mathcal{N}=3$ and $\mathcal{N}=4$ superconformal Abelian Chern-Simons theories in the projective superspace formalism. We also construct coupling terms among the gauge fields and matter hypermultiplets.
\end{abstract}

\footnotetext{
${ }^{1}$ masato.arai(at)utef.cvut.cz

${ }^{2}$ shin-s(at)kitasato-u.ac.jp
} 


\section{Introduction}

Chern-Simons theories in three dimensions have been attracted physicist's attention because of its importance in condensed matter and particle physics. Especially in the field of the latter, supersymmetric extensions of Chern-Simons theories have been intensively studied. It is known that three-dimensional $\mathcal{N}=2$ Chern-Simons-matter models admit (non)topological solitons due to the non-trivial Higgs potential determined by the $\mathcal{N}=2$ supersymmetry. The formulation of Chern-Simons-matter models with $\mathcal{N}=3$ supersymmetry is examined in [1]. The authors showed that the maximal supersymmetry of Chern-Simons-matter models in three dimensions with a single gauge field and no gravity is $\mathcal{N}=3$. Even though the $\mathcal{N}=3$ supersymmetry is the maximal one in that case, $\mathcal{N} \geq 4$ supersymmetries are possible for pure Chern-Simons theories [2, 3, 4] and quiver gauge theories.

Recently the low-energy effective theory of multiple M2-branes is proposed by Bagger, Lambert and Gustavsson (BLG model) [5, 6] which is based on the idea of the novel gauge group $\mathcal{A}_{4}$ constructed by 3 -algebras. Soon after the proposal, it is shown that the BLG model with $\mathcal{A}_{4}$ group is nothing but the $\mathcal{N}=8$ superconformal Chern-Simons-matter model with gauge group $S U(2) \times S U(2)$ with bi-fundamental matters [7]. Other Chern-Simons models with products of gauge groups and matters, such as $\mathcal{N}=4$ and $\mathcal{N}=5$ superconformal Chern-Simons-matter models [8], the $\mathcal{N}=6 U(N) \times U(N)$ model (ABJM model) [9] have also been constructed.

Besides these facts, manifestly supersymmetric formulations of Chern-Simons-matter models have been interesting topics. For example, Abelian and non-Abelian Chern-Simons-matter models in three-dimensional $\mathcal{N}=2$ superspace are constructed in [10, 11]. It is known that to incorporate manifest and off-shell $\mathcal{N} \geq 3$ supersymmetries (hence $\mathcal{N} \geq 2$ in four dimensions), the ordinary superspace approach is not suitable. The on-shell superfield formulation of the $\mathcal{N}=8$ BLG and the $\mathcal{N}=6$ ABJM models are found in [12. A good way to introduce the off-shell $\mathcal{N} \geq 3$ supersymmetries is to use the harmonic superspace approach [13, 14]. Pure (without matter) $\mathcal{N}=5,6$ Chern-Simons theories are studied in the framework of harmonic superspace [15, 16]. For the Chern-Simons theories with matter fields, a manifestly supersymmetric construction of the $\mathcal{N}=6$ ABJM model is investigated in the $\mathcal{N}=3$ harmonic superspace [17].

Another way to treat the off-shell extended supersymmetries is to use the projective superspace approach [18, 19, 20] which keeps $\mathcal{N}=2$ manifest supersymmetry in four dimensions. The two approaches have the relationship [21] and it is quite interesting to investigate the manifestly supersymmetric formulation of Chern-Simons-matter models in the projective superspace. Even more, it is possible to construct the action with manifest superconformal invariance in the projective superspace [22, 23]. In this paper, we study manifest $\mathcal{N}=3$ and $\mathcal{N}=4$ superconformal formulations of Chern-Simons-matter models in the projective superspaces. The analysis of this paper provides an alternative formulation of supersymmetric Chern-Simons theories other than the harmonic superspace approach. A projective superspace formulation of the BLG model is proposed in [24]. We will give a comment on this construction in the discussion.

The organization of this paper is as follows. In the next section, the $\mathcal{N}=3$ supersymmetric Chern-Simons-matter models in the $\mathcal{N}=2$ superspace is shown. In Section 3, we give a brief review of the three-dimensional $\mathcal{N}=3$ projective superspace formulation of superconformal theories. In Section 4, we formulate the Abelian Chern-Simons-matter models in $\mathcal{N}=3$ 
projective superspace approach and show that the proposed action correctly reproduces the result constructed in $\mathcal{N}=2$ superspace. Section 5 is devoted to the $\mathcal{N}=4$ generalization of the construction. Section 6 is conclusion and discussions where non-Abelian generalization is briefly discussed. Notations and conventions of $\mathcal{N}=2,3,4$ superspaces are given in Appendix A. Detail calculations of the solution to the projective superspace constraint are shown in Appendix B The anti-commutation relations among the gauge covariant derivatives are found in Appendix C.

\section{Chern-Simons-matter models in $\mathcal{N}=2$ superspace}

In this section, we briefly introduce the three-dimensional Chern-Simons-matter models in $\mathcal{N}=$ 2 superspace. It has been shown that Chern-Simons-matter models with a single gauge group have $\mathcal{N}=3$ maximal supersymmetry [1, 25] which will be enhanced to $\mathcal{N}>3$ superconformal symmetries when appropriate gauge groups and matters are added [8, 9].

Although our main interest is Abelian Chern-Simons-matter models, we start from nonAbelian gauge groups for generality. We first consider an $\mathcal{N}=2 U(N)$ Chern-Simons-matter model with level $k$, interacting with $N_{f}$ flavors. The model consists of the three-dimensional $\mathcal{N}=2$ vector superfield $V_{0}$ and the chiral, antichiral superfields $Q_{i}, \bar{Q}_{i}\left(i=1, \cdots N_{f}\right)$ representing the gauge field and matters respectively. The chiral and antichiral superfields which satisfy the conditions $\overline{\mathbb{D}}_{\alpha} Q_{i}=0$ and $\mathbb{D}_{\alpha} \bar{Q}_{i}=0$ are expanded as

$$
\begin{aligned}
& Q_{i}\left(x_{L}, \theta\right)=q_{i}\left(x_{L}\right)+\sqrt{2} \theta \psi_{q i}\left(x_{L}\right)+\theta^{2} F_{q i}\left(x_{L}\right), \\
& \bar{Q}_{i}\left(x_{R}, \bar{\theta}\right)=\bar{q}_{i}\left(x_{R}\right)-\sqrt{2} \bar{\theta} \bar{\psi}_{\bar{q} i}\left(x_{R}\right)-\bar{\theta}^{2} \bar{F}_{\bar{q} i}\left(x_{R}\right),
\end{aligned}
$$

where $\overline{\mathbb{D}}_{\alpha}$ and $\mathbb{D}_{\alpha}$ are the supercovariant derivatives in the $\mathcal{N}=2$ superspace, $x_{L}$ and $x_{R}$ are the chiral and antichiral coordinates defined in Appendix A. The vector superfield in the Wess-Zumino gauge is expanded as

$$
V_{0}(x, \theta, \bar{\theta})=2 i \theta \bar{\theta} \sigma(x)+2 \theta \gamma^{m} \bar{\theta} A_{m}(x)-\sqrt{2} i \bar{\theta}^{2} \theta \chi(x)+\sqrt{2} i \theta^{2} \bar{\theta} \bar{\chi}(x)+\theta^{2} \bar{\theta}^{2} D(x) .
$$

Here $A_{m}(m=0,1,2)$ is the gauge field, $\chi$ is the gaugino, $D$ is the auxiliary field and $\sigma$ is the real scalar. All the component fields in $V_{0}$ are in the adjoint representation of the gauge group. This is obtained by the dimensional reduction of the four-dimensional $\mathcal{N}=1$ vector superfield. Actually, $\sigma$ is the $A_{3}$ component of the four-dimensional gauge field. In order to write down the $\mathcal{N}=2$ supersymmetric action, we employ the trick proposed in [10] by introducing an auxiliary integration variable $t$ and express the Chern-Simons part as the integration of the exponentiated vector superfield. The action is given by

$$
S_{\mathrm{CSH}}^{\mathcal{N}=2}=\int d^{3} x \int d^{4} \theta\left\{-\frac{i k}{4 \pi} \int_{0}^{1} d t \operatorname{Tr}\left[V_{0} \overline{\mathbb{D}}^{\alpha}\left(e^{-t V_{0}} \mathbb{D}_{\alpha} e^{t V_{0}}\right)\right]+\sum_{i=1}^{N_{f}} \bar{Q}_{i} e^{V_{0}} Q_{i}\right\}
$$

where the symbol Tr is the gauge trace and $Q_{i}\left(\bar{Q}_{i}\right)$ are in the (anti)fundamental representation of the gauge group. The gauge trace is normalized as $\operatorname{Tr}\left(T^{a} T^{b}\right)=\delta^{a b}$ for the $U(N)$ generators 
$T^{a}$. The Chern-Simons level $k$ should be quantized to be integer valued for the gauge group $U(N)$. The action is gauge invariant under the following gauge transformation,

$$
e^{V_{0}} \longrightarrow e^{i \bar{\Lambda}} e^{V_{0}} e^{-i \Lambda}, \quad Q_{i} \rightarrow e^{i \Lambda} Q_{i}, \quad \bar{Q}_{i} \rightarrow e^{-i \bar{\Lambda}} \bar{Q}_{i},
$$

where $\Lambda, \bar{\Lambda}$ are gauge parameters satisfying the chiral, antichiral superfield conditions respectively. The second term in (2.3) is obtained just by the dimensional reduction of the fourdimensional matter kinetic term while the first term in (2.3) can not be obtained from the four dimensions. Note that this model is quantum mechanically conformal provided that there is no superpotential for $Q_{i}[26]$.

Next, we consider $\mathcal{N}=3$ Chern-Simons-matter theories. To construct the $\mathcal{N}=3$ supersymmetric action in $\mathcal{N}=2$ superfield formalism, one needs to introduce other chiral and antichiral multiplets $\Phi, \bar{\Phi}$ with adjoint representation of the gauge group. They are non-dynamical auxiliary fields. Combined with the vector superfield $V_{0}$, these form the $\mathcal{N}=4$ vector multiplet. The vector multiplet can couple to $\mathcal{N}=4$ hypermultiplets which are represented by pairs of chiral and antichiral multiplets $\left(S_{i}, T_{i}\right),\left(\bar{S}_{i}, \bar{T}_{i}\right)$ transforming in conjugate representations of the gauge group. The system including the Chern-Simons part and the matter part has only $\mathcal{N}=3$ supersymmetry since the Chern-Simons term breaks $\mathcal{N}=4$ supersymmetry down to $\mathcal{N}=3$. The $\mathcal{N}=3$ supersymmetric Chern-Simons action is given by [26]

$$
\begin{array}{r}
S_{\mathrm{CS}}^{\mathcal{N}=3}=\frac{-i k}{4 \pi} \int d^{3} x d^{4} \theta \int_{0}^{1} d t \operatorname{Tr}\left[V_{0} \overline{\mathbb{D}}^{\alpha}\left(e^{t V_{0}} \mathbb{D}_{\alpha} e^{-t V_{0}}\right)\right] \\
-\frac{k}{4 \pi} \int d^{3} x d^{2} \theta \operatorname{Tr} \Phi^{2}+\frac{k}{4 \pi} \int d^{3} x d^{2} \bar{\theta} \operatorname{Tr} \bar{\Phi}^{2}
\end{array}
$$

while the matter part is

$$
S_{\mathrm{H}}^{\mathcal{N}=3}=\int d^{3} x d^{4} \theta \sum_{i=1}^{N_{f}}\left(\bar{S}_{i} e^{V_{0}} S_{i}+T_{i} e^{-V_{0}} \bar{T}_{i}\right)+\sum_{i=1}^{N_{f}}\left[2 \int d^{3} x d^{2} \theta T_{i} \Phi S_{i}-2 \int d^{3} x d^{2} \bar{\theta} \bar{T}_{i} \bar{\Phi}_{S_{i}}\right]
$$

The action $S_{\mathrm{CS}}^{\mathcal{N}=3}+S_{\mathrm{H}}^{\mathcal{N}=3}$ keeps only $\mathcal{N}=2$ manifest supersymmetry but actually preserves $\mathcal{N}=3$ supersymmetry. This model is also quantum mechanically conformal.

Finally, let us take the Abelian limit of (2.5) for later convenience. In the Abelian case, we do not need the auxiliary $t$-integration. Therefore the $\mathcal{N}=3$ Abelian Chern-Simons action is given by

$$
S_{\mathrm{CS}}^{\mathcal{N}=3}=\frac{i k}{8 \pi} \int d^{3} x d^{4} \theta V_{0} \overline{\mathbb{D}}^{\alpha} \mathbb{D}_{\alpha} V_{0}-\frac{k}{4 \pi} \int d^{3} x d^{2} \theta \Phi^{2}+\frac{k}{4 \pi} \int d^{3} x d^{2} \bar{\theta} \bar{\Phi}^{2} .
$$

\section{Brief survey of projective superspace formalism}

In this section, we briefly review the basic ideas of the three-dimensional $\mathcal{N}=3$ superconformal projective superspace [22]. For those who are not familiar with the projective superspace approach, let us recall the ordinary $d=4, \mathcal{N}=1$ superfield formalism. The $d=4, \mathcal{N}=$ 1 superspace is parametrized by the space-time coordinate $x^{\mu}(\mu=0, \cdots, 3)$ and $S O(1,3)$ spinor coordinates $\theta_{\alpha}, \bar{\theta}_{\dot{\alpha}}$. As an explicit example, we consider a supersymmetric Lagrangian 
constructed by chiral superfields. A chiral superfield $\Phi$ is not a function of the full superspace, but a function of its subspace, called the chiral subspace. This subspace is defined by the constraint $\bar{D}_{\dot{\alpha}} \Phi=0$, where $\bar{D}_{\dot{\alpha}}=-\bar{\partial}_{\dot{\alpha}}-i\left(\theta \sigma^{\mu}\right)_{\dot{\alpha}} \partial_{\mu}$ and $\sigma^{\mu}$ are the four-dimensional sigma matrices. The supercovariant derivative $\bar{D}_{\dot{\alpha}}$ is used to define the chiral superfield whilst the other supercovariant derivative $D_{\alpha}=\partial_{\alpha}+i\left(\sigma^{\mu} \bar{\theta}\right)_{\alpha} \partial_{\mu}$ gives the integral measure to form the supersymmetric Lagrangian:

$$
\mathcal{L}=-\frac{D^{2}}{4} W(\Phi)+\text { h.c. }=\int d^{2} \theta W(\Phi)+\text { h.c. },
$$

where $W$ is a superpotential.

Analogously, we can construct a superconformal Lagrangian in the $\mathcal{N}=3$ projective superspace formalism in three dimensions. The $\mathcal{N}=3$ projective superspace consists of the ordinary $\mathcal{N}=3$ superspace $\mathbb{M}^{3 \mid 6}$ and the internal space $\mathbb{C} P^{1}$. They are parametrized by the super-coordinate $z^{M}=\left(x^{m}, \theta_{I}^{\alpha}\right)$ and $S U(2)_{R}$ complex isospinors $v^{i}, u^{i}$. Here $\alpha=1,2$ is the $S O(1,2) \sim S L(2, \mathbf{R})$ spinor and $I=1,2,3$ is the $S O(3)_{R} \sim S U(2)_{R}$ R-symmetry vector index respectively. We require that the two complex isospinors satisfy the following completeness relation,

$$
\delta_{j}^{i}=\frac{1}{(v, u)}\left(v^{i} u_{j}-v_{j} u^{i}\right), \quad(v, u) \equiv v^{i} u_{i}
$$

where $u_{i}$ is only restricted by the condition $(v, u) \neq 0$. We basically use the $S U(2)_{R}$ spinor indices $i, j=1,2$ rather than the $S O(3)_{R}$ vector indices. These are intertwined by the relation $\theta_{i j}^{\alpha}=\left(\tau_{I}\right)_{i j} \theta_{I}^{\alpha}$ where $\left(\tau_{I}\right)^{i}{ }_{j}$ are the Pauli matrices. The $S U(2)_{R}$ indices are raised and lowered by the anti-symmetric symbols $\varepsilon^{i j}, \varepsilon_{i j}$ such as $\theta^{i}=\varepsilon^{i j} \theta_{j}$. The basic convention of ordinary superspaces and the relation among $\mathcal{N}=3,4$ and $\mathcal{N}=2$ superspaces are presented in Appendix A.

The supercovariant derivatives in $\mathcal{N}=3$ superspace are defined by

$$
D_{\alpha}^{i j}=\frac{\partial}{\partial \theta_{i j}^{\alpha}}+i \theta_{i j}^{\beta} \partial_{\alpha \beta}, \quad \partial_{\alpha \beta} \equiv \gamma_{\alpha \beta}^{m} \partial_{m}
$$

These satisfy the following algebra:

$$
\left\{D_{\alpha}^{i j}, D_{\beta}^{k l}\right\}=-2 i \varepsilon^{i(k} \varepsilon^{l) j} \partial_{\alpha \beta} .
$$

Using the isospinors $v^{i}, u^{i}$ satisfying (3.2), we define the following set of supercovariant derivatives in the projective superspace:

$$
D_{\alpha}^{(2)}=v_{i} v_{j} D_{\alpha}^{i j}, \quad D_{\alpha}^{(0)}=\frac{1}{(v, u)} v_{i} u_{j} D_{\alpha}^{i j}, \quad D_{\alpha}^{(-2)}=\frac{1}{(v, u)^{2}} u_{i} u_{j} D_{\alpha}^{i j}
$$

where the superscripts on $D_{\alpha}^{(2)}, D_{\alpha}^{(0)}$ and $D_{\alpha}^{(-2)}$ indicate the degree of homogeneity in $v$ s. Among these covariant derivatives, $D_{\alpha}^{(2)}$ is used to define a superconformal projective multiplet. We define the superconformal projective multiplet $Q^{(n)}$ with weight $n \in \mathbb{Z}$, as a superfield being function of $z^{M}$ and $v^{i}$, by the following condition,

$$
D_{\alpha}^{(2)} Q^{(n)}=0
$$


which is an analogy to the chiral condition $\bar{D}_{\dot{\alpha}} \Phi=0$ in $d=4, \mathcal{N}=1$ superfield formalism. In addition, the superfield $Q^{(n)}$ should be holomorphic with respect to $v^{i}$ and homogeneous function of degree $n$,

$$
Q^{(n)}(z, c v)=c^{n} Q^{(n)}(z, v), \quad c \in \mathbb{C}^{*}
$$

The superconformal transformation of the superfield $Q^{(n)}$ is given by

$$
\delta Q^{(n)}=-\left(\xi-\Lambda^{(2)} \partial^{(-2)}\right) Q^{(n)}-n \Sigma Q^{(n)},
$$

where $\xi=\xi^{m} \partial_{m}+\xi_{I}^{\alpha} D_{\alpha}^{I}$ with $D_{\alpha}^{i j}=\left(\tau_{I}\right)^{i j} D_{\alpha}^{I}$ is the superconformal killing vector field and $\boldsymbol{\partial}^{(-2)}=\frac{1}{(v, u)} u^{i} \frac{\partial}{\partial v^{i}}$ is the differentiation with respect to the isospinor $v^{i}$. The parameters $\Lambda^{(2)}=v_{i} v_{j} \Lambda^{i j}$ and $\Sigma$ correspond to $S O(3)_{R}$ and the scale transformations respectively. Detailed explanation of the superconformal transformation is found in [22]. A conjugation which is consistent with the constraint (3.6) is called the smile conjugation. This is defined by

$$
\left.\breve{Q}^{(n)}(v) \equiv \overline{Q^{(n)}(v)}\right|_{\overline{v^{i}} \rightarrow-v_{i}},
$$

where the bar stands for the ordinary complex conjugation and the conjugation of $v^{i}$ is, more explicitly, $\overline{v^{1}} \rightarrow-v_{1}=v^{2}$ and $\overline{v^{2}} \rightarrow-v_{2}=-v^{1}$.

Now we construct the $\mathcal{N}=3$ superconformal invariant action. The supercovariant derivative $D_{\alpha}^{(2)}$ has been used to define the projective multiplet while the others $D_{\alpha}^{(0)}, D_{\alpha}^{(-2)}$ are used to form the Grassmann integral measure in an $\mathcal{N}=3$ superconformal action. The resultant action is given by [22]:

$$
S=\left.\frac{1}{8 \pi} \oint_{\gamma}(v, d v) \int d^{3} x\left(D^{(-2)}\right)^{2}\left(D^{(0)}\right)^{2} \mathcal{L}^{(2)}(z, v)\right|_{\theta=0}
$$

where $\mathcal{L}^{(2)}$ is an weight-2 real superconformal projective multiplet. Note that the action is formed so that sum of the degree of homogeneity in $v$ s is zero. We sometimes call $\mathcal{L}^{(2)}$ Lagrangian. The line integral is evaluated over a closed contour $\gamma$ in $\mathbb{C} P^{1}$. Along the contour, $u^{i}$ should satisfy $(v, u) \neq 0$. It is shown that the action (3.10) is $u$ independent and we can therefore choose $u_{i}=(1,0)$.

In the following, we rewrite the action (3.10) to the one in terms of $\mathcal{N}=2$ superspace and superfields. Without loss of generality, we can take the contour $\gamma$ in (3.10) such that it does not pass through the north pole $v^{i}=(0,1)$ in $\mathbb{C} P^{1}$. It is then useful to introduce a complex inhomogeneous coordinate $\zeta \in \mathbb{C}$ in the upper hemisphere (north chart) of $\mathbb{C} P^{1}$,

$$
v^{i}=v^{1}(1, \zeta), \quad \zeta \equiv \frac{v^{2}}{v^{1}}, \quad i=1,2 .
$$

We consider the projective multiplet in this chart. As we will see, this $\zeta$ is identified with the projective coordinate in the ordinary projective superspace formalism [18]. Using the coordinate $\zeta$, the covariant derivative $D_{\alpha}^{(2)}$ turns into the form

$$
\begin{aligned}
& D_{\alpha}^{(2)}=\left(v^{1}\right)^{2} D_{\alpha}^{[2]} \\
& D_{\alpha}^{[2]}(\zeta) \equiv-\overline{\mathbb{D}}_{\alpha}-2 \zeta D_{\alpha}^{12}+\zeta^{2} \mathbb{D}_{\alpha}
\end{aligned}
$$


where we have introduced the $\mathcal{N}=2$ supercovariant derivatives $\mathbb{D}_{\alpha}$ and $\overline{\mathbb{D}}_{\alpha}$ (see Appendix A). As discussed in 222, all the $v^{1}$ dependence of the superconformal projective multiplet $Q^{(n)}(z, v)$ can be factored out and a new superfield $Q^{[n]}(z, v) \propto Q^{(n)}(z, v)$ is defined. With the use of this fact and (3.12), we find that the constraint (3.6) becomes

$$
D_{\alpha}^{[2]}(\zeta) Q^{[n]}(z, \zeta)=0 .
$$

In general, $Q^{[n]}$ is expanded by power series in $\zeta$,

$$
Q^{[n]}(z, \zeta)=\sum_{k} \zeta^{k} Q_{k}(z)
$$

where $Q_{k}(z)$ are some ordinary $\mathcal{N}=3$ superfields subject to the constraints (3.13). Using the factorization of $v^{1}$, the projective multiplet $\mathcal{L}^{(2)}$ is rewritten as

$$
\mathcal{L}^{(2)}(z, v)=\left(v^{1}\right)^{2}(i \zeta) \mathcal{L}^{[2]}(z, \zeta)
$$

and the action (3.10) reduces to the following form,

$$
S=\left.\frac{1}{2 \pi i} \oint_{\gamma} \frac{d \zeta}{\zeta} \int d^{3} x d^{4} \theta \mathcal{L}^{[2]}(z, \zeta)\right|_{\theta_{12}=0}
$$

where we have used (3.12) and (3.13). The factor $i \zeta$ also appears in addition to $\left(v^{1}\right)^{2}$ for reality of $\mathcal{L}^{(2)}$ (see also $\mathcal{O}(-k, k)$ and tropical multiplets below). The expression (3.16) is analogous to the action in $d=4, \mathcal{N}=2$ ordinary projective superspace and is completely determined by the $\mathcal{N}=3$ superfields $Q_{k}(z)$ projected on the $\mathcal{N}=2$ superspace. Note that even though the integration in the action is carried out over the $\mathcal{N}=2$ superspace only, the action has $\mathcal{N}=3$ off-shell supersymmetry by construction.

We now give several examples of the projective superfields $Q^{(n)}$.

- $\mathcal{O}(k)$ and (ant)arctic multiplets

The weight- $n$ complex $\mathcal{O}(k)$ multiplet is defined to be holomorphic in the north chart of $\mathbb{C} P^{1}$,

$$
\Upsilon^{(n)}(z, v)=\left(v^{1}\right)^{n} \Upsilon^{[n]}(z, \zeta), \quad \Upsilon^{[n]}=\sum_{l=0}^{k} \zeta^{l} \Upsilon_{l}(z)
$$

The constraints (3.13) on the $\mathcal{N}=3$ component superfields are given by

$$
\begin{aligned}
& \overline{\mathbb{D}}_{\alpha} \Upsilon_{0}=0 \\
& \overline{\mathbb{D}}_{\alpha} \Upsilon_{1}+2 D_{\alpha}^{12} \Upsilon_{0}=0, \\
& \overline{\mathbb{D}}_{\alpha} \Upsilon_{l}+2 D_{\alpha}^{12} \Upsilon_{l-1}-\mathbb{D}_{\alpha} \Upsilon_{l-2}=0, \quad(2 \leq l \leq k), \\
& 2 D_{\alpha}^{12} \Upsilon_{k}-\mathbb{D}_{\alpha} \Upsilon_{k-1}=0, \\
& \mathbb{D}_{\alpha} \Upsilon_{k}=0 .
\end{aligned}
$$

The arctic multiplet is defined as the limit $k \rightarrow \infty$ of the complex $\mathcal{O}(k)$ multiplet and its smile conjugate is called the antarctic multiplet. 
- $\mathcal{O}(-k, k)$ and tropical multiplets

The real $\mathcal{O}(-k, k)$ multiplet with weight $n$ is defined as

$$
\begin{aligned}
& U^{(2 n)}(z, v)=\left(i v^{1} v^{2}\right)^{n} U^{[2 n]}(z, \zeta)=\left(v^{1}\right)^{2 n}(i \zeta)^{n} U^{[2 n]}(z, \zeta), \\
& U^{[2 n]}(z, \zeta)=\sum_{l=-k}^{k} \zeta^{l} U_{l}(z), \quad \bar{U}_{l}=(-1)^{l} U_{-l} .
\end{aligned}
$$

The constraints (3.13) on the $\mathcal{N}=3$ component superfields are given by

$$
\begin{aligned}
& \overline{\mathbb{D}}_{\alpha} U_{-k}=0, \\
& \overline{\mathbb{D}}_{\alpha} U_{-k+1}+2 D_{\alpha}^{12} U_{-k}=0, \\
& \overline{\mathbb{D}}_{\alpha} U_{l}+2 D_{\alpha}^{12} U_{l-1}-\mathbb{D}_{\alpha} U_{l-2}=0, \quad(-k+2 \leq l \leq k), \\
& 2 D_{\alpha}^{12} U_{k}-\mathbb{D}_{\alpha} U_{k-1}=0, \\
& \mathbb{D}_{\alpha} U_{k}=0 .
\end{aligned}
$$

The tropical multiplet is defined as the limit $k \rightarrow \infty$ of the $\mathcal{O}(-k, k)$ multiplet.

\section{$4 \mathcal{N}=3$ superconformal Chern-Simons-matter models}

In this section, we construct $\mathcal{N}=3$ supersymmetric Chern-Simons-matter actions with Abelian gauge group in the projective superspace.

\subsection{Chern-Simons term}

We expect that the supersymmetric Chern-Simons term is naively given by the product of a (super)gauge field strength and a gauge potential, which is inspired by the result in fivedimensional projective superspace [28, 29]. The gauge potential is given by the real tropical multiplet $\mathcal{V}^{(0)}(z, v)$ with weight 0 . For the gauge field strength, we assume that it is given by a real $\mathcal{O}(-1,1)$ multiplet $G^{(2)}(z, v)$ with weight 2 . Then we propose that the weight-2 superconformal projective superfield in the action is given by

$$
\mathcal{L}^{(2)}=\frac{k}{8 \pi} \mathcal{V}^{(0)}(z, v) G^{(2)}(z, v) .
$$

Since the tropical multiplet is weight 0 , there is no overall $v^{1}$ dependence, $\mathcal{V}^{(0)}(z, v)=\mathcal{V}^{[0]}(z, \zeta)$. It is expanded with respect to $\zeta$ as

$$
\mathcal{V}^{[0]}(z, \zeta)=\sum_{n=-\infty}^{\infty} \zeta^{n} V_{n}(\zeta)
$$

The tropical multiplet transforms under the $U(1)$ gauge transformation:

$$
\delta \mathcal{V}^{[0]}=i\left(\bar{\Lambda}^{[0]}-\Lambda^{[0]}\right), \quad \Lambda^{[0]}=\sum_{n=0}^{\infty} \zeta^{n} \lambda_{n}, \quad \bar{\Lambda}^{[0]}=\sum_{n=0}^{\infty}(-1 / \zeta)^{n} \bar{\lambda}_{n}
$$


where $\Lambda^{[0]}(z, \zeta)$ and $\bar{\Lambda}^{[0]}(z, \zeta)$ are gauge parameters in the weight-0 arctic and antarctic multiplets $\Lambda^{(0)}(z, \zeta)$ and $\bar{\Lambda}^{(0)}(z, \zeta)$ respectively. The gauge transformations of the real tropical multiplet in terms of $\zeta$-expansion coefficients are

$$
\delta V_{0}=i\left(\bar{\lambda}_{0}-\lambda_{0}\right), \quad \delta V_{n}=-i \lambda_{n}
$$

By the gauge transformation, we can make the tropical multiplet be a real $\mathcal{O}(-1,1)$ multiplet form (Lindström-Roček gauge) [20],

$$
\mathcal{V}^{[0]}=\frac{1}{\zeta} V_{-1}+V_{0}+\zeta V_{1} .
$$

The weight-2 $\mathcal{O}(-1,1)$ multiplet becomes $G^{(2)}=\left(v^{1}\right)^{2}(i \zeta) G^{[2]}(z, \zeta)$ where $G^{[2]}$ is expanded as

$$
G^{[2]}(z, \zeta)=\frac{i}{\zeta} \Phi+L+i \zeta \bar{\Phi}
$$

We assume that $G^{[2]}$ is invariant under the $U(1)$ gauge transformation. Indeed, as we will see in below, the components in $G^{[2]}$ are written in terms of the components in $\mathcal{V}^{[0]}$ as the gauge invariant forms.

As we have discussed in the previous section, the constraint (3.13) is interpreted as the constraints on the $\mathcal{N}=3$ component superfields. Since the action is totally expressed in terms of $\mathcal{N}=3$ component superfields projected on the $\mathcal{N}=2$ superspace, we are interested in the constraints in the $\mathcal{N}=2$ subsuperspace. From the constraints (3.18) and (3.20), we can find the constraints for the component superfields. For $\mathcal{V}^{(0)}$, since this is the tropical multiplet, $V_{0} \mid$ is a real superfield and other fields are unconstrained. Here the symbol "|" means the $\mathcal{N}=2$ projection of $\mathcal{N}=3$ superfield. For the $\mathcal{O}(-1,1)$ multiplet $G^{[2]}$, we have constraints

$$
\begin{aligned}
& \overline{\mathbb{D}}_{\alpha} \Phi \mid=0 \\
& \overline{\mathbb{D}}^{2} L\left|=\mathbb{D}^{2} L\right|=0, \\
& \mathbb{D}_{\alpha} \bar{\Phi}=0
\end{aligned}
$$

These conditions imply that $\Phi \mid$ and $\bar{\Phi} \mid$ are the $\mathcal{N}=2$ chiral and antichiral superfields while $L \mid$ is the real linear superfield. Finally, for the gauge parameters $\Lambda^{[0]}(z, \zeta)$ and $\bar{\Lambda}^{[0]}(z, \zeta)$, we have

$$
\begin{aligned}
& \overline{\mathbb{D}}_{\alpha} \lambda_{0}\left|=\mathbb{D}_{\alpha} \bar{\lambda}_{0}\right|=0, \\
& \overline{\mathbb{D}}^{2} \lambda_{1}\left|=\mathbb{D}^{2} \bar{\lambda}_{1}\right|=0,
\end{aligned}
$$

and other fields are unconstrained. Therefore $\lambda_{0} \mid$ and $\bar{\lambda}_{0} \mid$ are $\mathcal{N}=2$ (anti)chiral while $\lambda_{1} \mid$ and $\bar{\lambda}_{1} \mid$ are $\mathcal{N}=2$ (anti)linear superfields. In the following we omit the symbol "|" and consider the component superfields in $\mathcal{N}=2$ superspace.

After fixing $u$ to $u_{i}=(1,0)$, the action (3.10) with the Lagrangian (4.1) reduces to the following form,

$$
S_{\mathrm{CS}}=\frac{k}{8 \pi} \oint_{\gamma} \frac{d \zeta}{2 \pi i \zeta} \int d^{3} x d^{4} \theta \mathcal{V}^{[0]}(z, \zeta) G^{[2]}(z, \zeta)
$$


The component superfields $L, \Phi$ and $\bar{\Phi}$ should be expressed in terms of $V_{n}$ and they satisfy the constraints (4.7). We find that the solutions to the constraints are given by

$$
L=i \overline{\mathbb{D}}^{\alpha} \mathbb{D}_{\alpha} V_{0}, \quad \Phi=\frac{i}{8} \overline{\mathbb{D}}^{\alpha} \overline{\mathbb{D}}_{\alpha} V_{1}, \quad \bar{\Phi}=-\frac{i}{8} \mathbb{D}^{\alpha} \mathbb{D}_{\alpha} V_{-1}
$$

One can confirm that all these expressions are gauge invariant by using the relations (4.8). This fact is consistent with our assumption. The solutions (4.10) are also expressed as the integral of the tropical multiplet over the isospinor space:

$$
\begin{aligned}
G^{(2)}(w)=-\frac{1}{8 \pi i} & \oint_{\gamma}(v, d v)\left\{-\frac{i}{2}(w, v)^{2} D^{(-2) \alpha} D_{\alpha}^{(-2)}\right. \\
& \left.+8 \frac{(w, v)(w, u)}{(v, u)} D^{(-2) \alpha} D_{\alpha}^{(0)}+2 i \frac{(w, u)^{2}}{(v, u)^{2}} D^{(0) \alpha} D_{\alpha}^{(0)}\right\} \mathcal{V}^{(0)}(v),
\end{aligned}
$$

This closed form expression is obtained in the context of three-dimensional supergravity in the projective superspace [23] 3. After fixing $u=w$ and using the completeness relation of the isospinor, we find that (4.11) is also expressed as

$$
G^{(2)}(w)=-\frac{1}{8 \pi i}\left(D^{(2)}(w)\right)^{2} \oint_{\gamma} \frac{(v, d v)}{(v, w)} \mathcal{V}^{(0)}(v) .
$$

The detailed calculations are found in Appendix B.

Next, we examine the gauge invariance of the action. Since the action (3.10) is completely equivalent to the expression (3.16) and the gauge variation $\delta \mathcal{V}^{[0]}$ is the tropical multiplet with weight 0 , it is sufficient to show the gauge invariance of (4.9) rather than its manifest $\mathcal{N}=3$ action (3.10). Substituting the expressions (4.4) and (4.6) into the gauge variation of the action $\delta S_{\mathrm{CS}}$ and integrating it with respect to $\zeta$, we find

$$
\delta S_{\mathrm{CS}}=\frac{k}{8 \pi} \int d^{3} x d^{4} \theta\left[\bar{\lambda}_{1} \bar{\Phi}+i\left(\bar{\lambda}_{0}-\lambda_{0}\right) L+\lambda_{1} \Phi\right] .
$$

This expression is vanishing, taking account of the constraints (4.7), (4.8) and the relations $d^{2} \theta=-\mathbb{D}^{\alpha} \mathbb{D}_{\alpha} / 4, d^{2} \bar{\theta}=-\overline{\mathbb{D}}^{\alpha} \overline{\mathbb{D}}_{\alpha} / 4$ in the space-time integration. Therefore $S_{\mathrm{CS}}$ is gauge invariant. Note that the higher order components in $\zeta^{n}$ are dropped in the expression due to the $\zeta$-integration.

To see the action in terms of $\mathcal{N}=2$ superfields, we substitute the solution (4.10) into the action (4.9) and find

$$
\begin{aligned}
S_{\mathrm{CS}}= & \frac{k}{8 \pi} \int d^{3} x d^{4} \theta\left(i V_{1} \Phi+i V_{0} \overline{\mathbb{D}}^{\alpha} \mathbb{D}_{\alpha} V_{0}+i V_{-1} \bar{\Phi}\right) \\
= & \frac{i k}{8 \pi} \int d^{3} x d^{4} \theta V_{0} \overline{\mathbb{D}}^{\alpha} \mathbb{D}_{\alpha} V_{0} \\
& \quad+\frac{i k}{8 \pi} \int d^{3} x d^{2} \theta\left(-\frac{\overline{\mathbb{D}}^{\alpha} \overline{\mathbb{D}}_{\alpha}}{4} V_{1}\right) \Phi+\frac{i k}{8 \pi} \int d^{3} x d^{2} \bar{\theta}\left(-\frac{\mathbb{D}^{\alpha} \mathbb{D}_{\alpha}}{4} V_{-1}\right) \bar{\Phi} \\
= & \frac{i k}{8 \pi} \int d^{3} x d^{4} \theta V_{0} \overline{\mathbb{D}}^{\alpha} \mathbb{D}_{\alpha} V_{0}-\frac{k}{4 \pi} \int d^{3} x d^{2} \theta \Phi^{2}+\frac{k}{4 \pi} \int d^{3} x d^{2} \bar{\theta} \bar{\Phi}^{2} .
\end{aligned}
$$

This is equivalent to the expression given in (2.7). Therefore the Lagrangian (4.1) correctly reproduces the $\mathcal{N}=3$ Chern-Simons action in $\mathcal{N}=2$ superfield formalism.

\footnotetext{
${ }^{3}$ We have slightly changed the coefficients to make it be consistent with our convention.
} 


\subsection{Matter part}

It is known that the matter hypermultiplet is embedded into the ant(arctic) multiplet. We consider the weight-1 arctic multiplet $\Upsilon^{(1)}$ as the matter part of the $\mathcal{N}=3$ Chern-Simonsmatter model. The Lagrangian of the free (ant)arctic multiplets is given by

$$
\mathcal{L}^{(2)}=i \breve{\Upsilon}^{(1)} \Upsilon^{(1)}=i\left(v^{1}\right)^{2} \zeta \bar{\Upsilon}^{[1]} \Upsilon^{[1]}
$$

This is easily expanded into the $\mathcal{N}=3$ component superfields. Substituting the expansion (3.17) into the action (3.16) and performing the $\zeta$-integration, we find

$$
S_{\text {free }}=\int d^{3} x d^{4} \theta \sum_{l=0}^{\infty}(-1)^{l} \bar{\Upsilon}_{l} \Upsilon_{l}
$$

According to the constraints (3.18) ) on the $\mathcal{N}=2$ projected superfields, only $\Upsilon_{0}\left(\bar{\Upsilon}_{0}\right)$ and $\Upsilon_{1}$ $\left(\bar{\Upsilon}_{1}\right)$ are dynamical fields and the others are auxiliary fields, which are integrated out with their equations of motion. The resulting action describes the four-dimensional $\mathcal{N}=2$ massless free hypermultiplet dimensionally reduced to three dimensions.

We now introduce a gauge interaction. We consider the action of the (ant)arctic multiplets coupled to the Abelian gauge field. Following the ordinary projective superfield approach in four dimensions [30], the Lagrangian is given by

$$
\mathcal{L}^{(2)}=i \breve{\Upsilon}^{(1)} \exp \left(\mathcal{V}^{(0)}\right) \Upsilon^{(1)}=i\left(v^{1}\right)^{2} \zeta \bar{\Upsilon}^{[1]} \exp \left(\mathcal{V}^{[0]}\right) \Upsilon^{[1]}
$$

The action (3.16) for this Lagrangian is given by

$$
S_{\text {matter }}=\int d^{3} x d^{4} \theta \oint_{\gamma} \frac{d \zeta}{2 \pi i \zeta} \bar{\Upsilon}^{[1]} e^{\mathcal{V}^{[0]}} \Upsilon^{[1]}
$$

It is easy to confirm that the action is invariant under the following $U(1)$ gauge transformations

$$
\Upsilon^{[1] \prime}=e^{i \Lambda^{[0]}} \Upsilon^{[1]}, \quad \bar{\Upsilon}^{[1] \prime}=e^{-i \bar{\Lambda}^{[0]}} \bar{\Upsilon}^{[1]}, \quad\left(e^{\mathcal{V}^{[0]}}\right)^{\prime}=e^{i \bar{\Lambda}^{[0]}} e^{\mathcal{V}^{[0]}} e^{-i \Lambda^{[0]}} .
$$

We write down the action (4.18) in terms of $\mathcal{N}=2$ superfields. In the Abelian case, it is always possible to split the tropical multiplet $\mathcal{V}^{[0]}$ into the arctic $\mathcal{V}_{+}$and the antarctic $\mathcal{V}_{-}$pieces,

$$
e^{\mathcal{V}^{[0]}}=e^{\mathcal{V}_{+}} e^{\mathcal{V}_{-}}
$$

where we have defined

$$
\mathcal{V}_{+}=\frac{1}{2} V_{0}+\sum_{n=1}^{\infty} \zeta^{n} V_{n}, \quad \mathcal{V}_{-}=\frac{1}{2} V_{0}+\sum_{n=1}^{\infty} \zeta^{-n} V_{-n}
$$

In the following, we employ the Lindström-Roček gauge so that the tropical multiplet $\mathcal{V}^{[0]}$ takes the $\mathcal{O}(-1,1)$ multiplet form. We define the gauge supercovariant derivative as

$$
\begin{aligned}
\mathcal{D}_{\alpha}^{[2]} & =e^{-q \mathcal{V}_{-}} D_{\alpha}^{[2]} e^{q \mathcal{V}_{-}}=e^{q \mathcal{V}_{+}} D_{\alpha}^{[2]} e^{-q \mathcal{V}_{+}} \\
& \equiv-\overline{\mathcal{D}}_{\alpha}-2 \zeta \mathcal{D}_{\alpha}^{12}+\zeta^{2} \mathcal{D}_{\alpha},
\end{aligned}
$$


where $q$ is the $U(1)$ charge of associated fields and

$$
\begin{aligned}
\mathcal{D}_{\alpha} & =\mathbb{D}_{\alpha}+\frac{q}{2} \mathbb{D}_{\alpha} V_{0} \\
\mathcal{D}_{\alpha}^{12} & =D_{\alpha}^{12}-\frac{q}{2}\left(\mathbb{D}_{\alpha} V_{-1}-D_{\alpha}^{12} V_{0}\right) \\
\overline{\mathcal{D}}_{\alpha} & =\overline{\mathbb{D}}_{\alpha}+\frac{q}{2}\left(\overline{\mathbb{D}}_{\alpha} V_{0}+4 D_{\alpha}^{12} V_{-1}\right) .
\end{aligned}
$$

The second equality in the first line in $(4.22)$ holds because of $D_{\alpha}^{[2]} e^{\mathcal{V}^{[0]}}=0$. The anticommutation relations of these supercovariant derivatives are found in Appendix C. The action (4.18) is rewritten as

$$
S_{\text {matter }}=\int d^{3} x d^{4} \theta \oint_{\gamma} \frac{d \zeta}{2 \pi i \zeta} \tilde{\Upsilon}^{[1]} \tilde{\Upsilon}^{[1]}
$$

where we have defined

$$
\begin{aligned}
& \tilde{\Upsilon}^{[1]}=e^{\mathcal{V}_{+}} \Upsilon^{[1]}=\sum_{n=0}^{\infty} \zeta^{n} \tilde{\Upsilon}_{n}, \\
& \tilde{\widetilde{\Upsilon}}^{[1]}=e^{\mathcal{V}_{-}} \bar{\Upsilon}^{[1]}=\sum_{n=0}^{\infty}\left(\frac{-1}{\zeta}\right)^{n} \tilde{\widetilde{\Upsilon}}_{n} .
\end{aligned}
$$

We can show that these superfields satisfy the gauge covariantized projective constraints $\mathcal{D}_{\alpha}^{[2]} \tilde{\Upsilon}^{[1]}=$ $\mathcal{D}_{\alpha}^{[2]} \tilde{\Upsilon}^{[1]}=0$ and give the gauge covariant (ant)arctic multiplets. The constraints on the component superfields are found to be

$$
\begin{aligned}
& \overline{\mathcal{D}}_{\alpha} \tilde{\Upsilon}_{0}=0, \\
& \overline{\mathcal{D}}_{\alpha} \tilde{\Upsilon}_{1}+2 \mathcal{D}_{\alpha}^{12} \tilde{\Upsilon}_{0}=0, \\
& \overline{\mathcal{D}}_{\alpha} \tilde{\Upsilon}_{l}+2 \mathcal{D}_{\alpha}^{12} \tilde{\Upsilon}_{l-1}-\mathcal{D}_{\alpha} \tilde{\Upsilon}_{l-2}=0, \quad(l \geq 2), \\
& \mathcal{D}_{\alpha} \tilde{\bar{\Upsilon}}_{0}=0, \\
& \mathcal{D}_{\alpha} \tilde{\widetilde{\Upsilon}}_{1}-2 \mathcal{D}_{\alpha}^{12} \tilde{\widetilde{\Upsilon}}_{0}=0, \\
& \mathcal{D}_{\alpha} \tilde{\widetilde{\Upsilon}}_{l-2}+2 \mathcal{D}_{\alpha}^{12} \tilde{\widetilde{\Upsilon}}_{l-1}-\mathcal{D}_{\alpha} \tilde{\widetilde{\Upsilon}}_{l}=0, \quad(l \geq 2) .
\end{aligned}
$$

With the use of the commutation relations (C.1) and (3.20), the constraints (4.27) and (4.30) turn out to be

$$
\overline{\mathcal{D}}^{2} \tilde{\Upsilon}_{1}=\overline{\mathbb{D}}^{2} V_{1} \tilde{\Upsilon}_{0}, \quad \mathcal{D}^{2} \tilde{\bar{\Upsilon}}_{1}=-\mathbb{D}^{2} V_{-1} \tilde{\bar{\Upsilon}}_{0}
$$

The matter action (4.24) is now expanded as

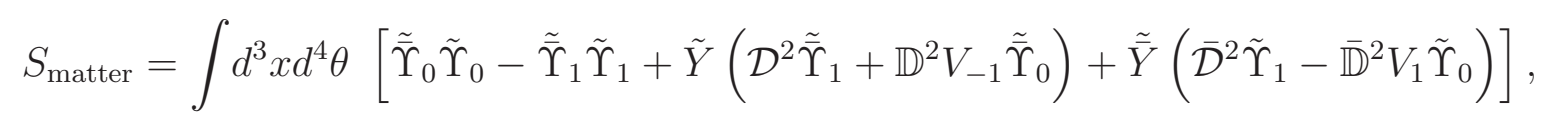

where we have integrated out the auxiliary fields $\tilde{\Upsilon}_{l}, \tilde{\bar{\Upsilon}}_{l}(l \geq 2)$. We have also introduced the Lagrange multipliers $\tilde{Y}, \tilde{\bar{Y}}$ which are related to the non-covariantized Lagrange multipliers $Y$ 
and $\bar{Y}$ through the relation $\tilde{Y}=e^{-\frac{1}{2} V_{0}} Y, \tilde{\bar{Y}}=e^{-\frac{1}{2} V_{0}} \bar{Y}$. Their gauge transformations are given by $Y \rightarrow e^{i \bar{\lambda}_{0}} Y, \bar{Y} \rightarrow e^{-i \lambda_{0}} \bar{Y}$. After integrating out $\tilde{\widetilde{\Upsilon}}_{1}, \tilde{\Upsilon}_{1}$ and using the constraints (4.26), (4.29), the solutions (4.10), and the anticommutation relations of the supercovariant derivatives (C.1), the action becomes

$$
S_{\text {matter }}=\int d^{3} x d^{4} \theta\left[\bar{S} e^{V_{0}} S+T e^{-V_{0}} \bar{T}\right]+2 \int d^{3} x d^{2} \theta T \Phi S-2 \int d^{3} x d^{2} \bar{\theta} \bar{T} \bar{\Phi} \bar{S} .
$$

Here we have relabeled $S=\Upsilon_{0}, \bar{S}=\bar{\Upsilon}_{0}$ and have defined

$$
\tilde{\bar{T}}=e^{-\frac{1}{2} V_{0}} \bar{T}=i \mathcal{D}^{2} \tilde{Y}, \quad \tilde{T}=e^{-\frac{1}{2} V_{0}} T=i \overline{\mathcal{D}}^{2} \tilde{\bar{Y}} .
$$

The gauge transformations of $\bar{T}, T$ are the same as the ones for the Lagrange multipliers $Y, \bar{Y}$. The action (4.34) agrees with (2.6) with $N_{f}=1$. Generalization to the arbitrary number of flavours $N_{f}$ is straightforward.

\section{$5 \mathcal{N}=4$ superconformal Chern-Simons theories}

In this section, we generalize the $\mathcal{N}=3$ construction discussed in the previous section to $\mathcal{N}=4$ cases. The $\mathcal{N}=4$ superspace $\mathbb{M}^{3 \mid 8}$ is parametrized by the super-coordinates $z^{M}=\left(x^{m}, \theta_{i j}^{\alpha}\right)$, where $i=1,2$ and $\bar{j}=1,2$ are indices for the $S U(2)_{L} \times S U(2)_{R}$ subgroup of $S O(4)_{R}$. These indices are raised and lowered by the antisymmetric matrices $\varepsilon^{i j}, \varepsilon^{\overline{i j}}$ and so on. To incorporate the two $S U(2)$ symmetries, we introduce a pair of $\mathbb{C} P^{1}$, namely, the mirror projective spaces $\mathbb{C} P_{L}^{1} \times \mathbb{C} P_{R}^{1}[22]$. As a result, the $\mathcal{N}=4$ projective superspace is given by $\mathbb{M}^{3 \mid 8} \times \mathbb{C} P_{L}^{1} \times \mathbb{C} P_{R}^{1}$. The mirror projective spaces $\mathbb{C} P_{L}^{1} \times \mathbb{C} P_{R}^{1}$ are parametrized by the homogeneous complex coordinates $v_{L}=\left(v^{i}\right), v_{R}=\left(v^{\bar{i}}\right)$. As in the case of $\mathcal{N}=3$, they are supplemented by $u_{i}, u_{\bar{k}}$ and satisfy the similar completeness relations as in (3.2).

The supercovariant derivatives in $\mathcal{N}=4$ superspace are given by

$$
D_{\alpha}^{i \bar{j}}=\frac{\partial}{\partial \theta_{i \bar{j}}^{\alpha}}+i \theta_{i \bar{j}}^{\beta} \partial_{\alpha \beta}
$$

They satisfy the following algebra

$$
\left\{D_{\alpha}^{i \bar{j}}, D_{\beta}^{k \bar{l}}\right\}=2 i \varepsilon^{i k} \varepsilon^{\bar{j} \bar{l}} \partial_{\alpha \beta} .
$$

As in the $\mathcal{N}=3$ case, we define the following covariant derivatives:

$$
D_{\alpha}^{(1) \bar{k}}=v_{i} D_{\alpha}^{i \bar{k}}, \quad D_{\alpha}^{(-1) \bar{k}}=\frac{1}{\left(v_{L}, u_{L}\right)} u_{i} D_{\alpha}^{i \bar{k}}
$$

and

$$
D_{\alpha}^{(1) i}=v_{\bar{k}} D_{\alpha}^{i \bar{k}}, \quad D_{\alpha}^{(-1) i}=\frac{1}{\left(v_{R}, u_{R}\right)} u_{\bar{k}} D_{\alpha}^{i \bar{k}}
$$

The covariant derivatives $D_{\alpha}^{(1) \bar{k}}, D_{\alpha}^{(-1) \bar{k}}$ satisfy the algebras,

$$
\begin{aligned}
& \left\{D_{\alpha}^{(1) \bar{k}}, D_{\beta}^{(1) \bar{l}}\right\}=\left\{D_{\alpha}^{(-1) \bar{k}}, D_{\beta}^{(-1) \bar{l}}\right\}=0, \\
& \left\{D_{\alpha}^{(1) \bar{k}}, D_{\beta}^{(-1) \bar{l}}\right\}=-2 i \varepsilon^{\bar{k}} \bar{l} \partial_{\alpha \beta} .
\end{aligned}
$$


The other covariant derivatives $D_{\alpha}^{(1) i}, D_{\alpha}^{(-1) i}$ satisfy the similar algebras. In the $\mathcal{N}=4$ case, we can introduce the left and right weight- $n$ projective multiplets associated with each $\mathbb{C} P^{1}$. They are defined by

$$
\begin{aligned}
& D_{\alpha}^{(1) \bar{k}} Q_{L}^{(n)}\left(v_{L}\right)=0, \\
& D_{\alpha}^{(1) i} Q_{R}^{(n)}\left(v_{R}\right)=0 .
\end{aligned}
$$

Each projective multiplet $Q_{L}^{(n)}, Q_{R}^{(n)}$ satisfies the relation (3.7). The $\mathcal{N}=4$ superconformal transformations of the left and right projective superfields are given by

$$
\begin{aligned}
& \delta Q_{L}^{(n)}=-\left(\xi-\Lambda_{L}^{(2)} \partial_{L}^{(-2)}\right) Q_{L}^{(n)}-n \Sigma_{L} Q_{L}^{(n)}, \\
& \delta Q_{R}^{(n)}=-\left(\xi-\Lambda_{R}^{(2)} \boldsymbol{\partial}_{R}^{(-2)}\right) Q_{R}^{(n)}-n \Sigma_{R} Q_{R}^{(n)}
\end{aligned}
$$

where $\xi$ is the superconformal Killing vector field, $\Lambda_{L, R}, \Sigma_{L, R}$ and $\boldsymbol{\partial}_{L, R}^{(-2)}$ are defined as in the same way in $\mathcal{N}=3$ case. See [22] for details.

Following the previous section, we introduce the complex inhomogeneous coordinate $\zeta_{L}$ in the left part as

$$
v^{i}=v^{1}\left(1, \zeta_{L}\right), \quad \zeta_{L}=\frac{v^{2}}{v^{1}}
$$

The covariant derivative becomes

$$
D_{\alpha}^{(1) \bar{k}}=v^{1} D_{\alpha}^{[1] \bar{k}}, \quad D_{\alpha}^{[1] \bar{k}} \equiv D_{\alpha}^{2 \bar{k}}-\zeta D_{\alpha}^{1 \bar{k}} .
$$

The $v^{1}$ dependencies of the projective superfields can be factored out and one can define $Q_{L}^{[n]} \propto$ $Q_{L}^{(n)}$ that satisfies

$$
D_{\alpha}^{[1] \bar{k}}(\zeta) Q_{L}^{[n]}=0
$$

Then, the left projective superfield $Q_{L}^{[n]}$ is expanded as

$$
Q_{L}^{[n]}\left(z, \zeta_{L}\right)=\sum_{k} \zeta_{L}^{k} Q_{k}(z)
$$

where $Q_{k}(z)$ are $\mathcal{N}=4$ superfields subject to the constraint (5.6). Similar definitions hold in the right part.

The $\mathcal{N}=4$ superconformal invariant action is given by

$$
S=\left.\frac{1}{2 \pi} \oint_{\gamma_{L}}\left(v_{L}, d v_{L}\right) \int d^{3} x D_{L}^{(-4)} \mathcal{L}_{L}^{(2)}\left(z, v_{L}\right)\right|_{\theta=0}+\left.\frac{1}{2 \pi} \oint_{\gamma_{R}}\left(v_{R}, d v_{R}\right) \int d^{3} x D_{R}^{(-4)} \mathcal{L}_{R}^{(2)}\left(z, v_{R}\right)\right|_{\theta=0},
$$

where $\mathcal{L}_{L}^{(2)}\left(\mathcal{L}_{R}^{(2)}\right)$ is the weight-2 left (right) projective multiplet and we have defined

$$
\begin{array}{ll}
D_{L}^{(-4)}=\frac{1}{48} D^{(-2) \bar{k} \bar{l}} D_{\bar{k} \bar{l}}^{(-2)}, & D_{\bar{k} \bar{l}}^{(-2)}=D_{\bar{k}}^{(-1) \alpha} D_{\alpha \bar{l}}^{(-1)} \\
D_{R}^{(-4)}=\frac{1}{48} D^{(-2) i j} D_{i j}^{(-2)}, & D_{i j}^{(-2)}=D_{i}^{(-1) \alpha} D_{\alpha j}^{(-1)}
\end{array}
$$


The contour $\gamma_{L}\left(\gamma_{R}\right)$ is chosen such that the path goes the outside of the north pole in $\mathbb{C} P_{L}^{1}$ $\left(\mathbb{C} P_{R}^{1}\right)$. After fixing $u_{i}=(1,0), u_{\bar{k}}=(1,0)$ in $\mathbb{C} P_{L}^{1}$ and $\mathbb{C} P_{R}^{1}$, the action can be rewritten as the one in $\mathcal{N}=2$ superspace:

$$
S=\left.\frac{1}{2 \pi i} \oint_{\gamma_{L}} \frac{d \zeta_{L}}{\zeta_{L}} \int d^{3} x d^{4} \theta \mathcal{L}_{L}^{[2]}\left(z, \zeta_{L}\right)\right|_{\theta_{\perp}=0}+\left.\frac{1}{2 \pi i} \oint_{\gamma_{R}} \frac{d \zeta_{R}}{\zeta_{R}} \int d^{3} x d^{4} \theta \mathcal{L}_{R}^{[2]}\left(z, \zeta_{R}\right)\right|_{\theta_{\perp}=0}
$$

where the symbol $\left.\right|_{\theta_{\perp}=0}$ means that the superfields are projected on the $\mathcal{N}=2$ superspace.

Classification of multiplets are similar to the $\mathcal{N}=3$ case. Since the left and right parts have almost the same structure, let us focus on the left part for the moment. A complex $\mathcal{O}(k)$ multiplet and a real $\mathcal{O}(-k, k)$ multiplet are defined as (3.17) and (3.19), respectively. Constraints for the components of a complex $\mathcal{O}(k)$ multiplet $\Upsilon^{[n]}=\sum_{l=0}^{k} \Upsilon_{l} \zeta^{l}$ are given by

$$
\begin{aligned}
& \overline{\mathbb{D}}_{\alpha} \Upsilon_{0}=D_{\alpha}^{2 \overline{1}} \Upsilon_{0}=0, \\
& \overline{\mathbb{D}}_{\alpha} \Upsilon_{l}=-D_{\alpha}^{1 \overline{2}} \Upsilon_{l-1}, \quad D_{\alpha}^{2 \overline{1}} \Upsilon_{l}=\mathbb{D}_{\alpha} \Upsilon_{l-1}, \quad(1 \leq l \leq k), \\
& \mathbb{D}_{\alpha} \Upsilon_{k}=D_{\alpha}^{1 \overline{2}} \Upsilon_{k}=0,
\end{aligned}
$$

while those on a real $\mathcal{O}(-k, k)$ multiplet $U^{[2 n]}=\sum_{l=-k}^{k} U_{l} \zeta^{l}$ are

$$
\begin{aligned}
& \overline{\mathbb{D}}_{\alpha} U_{-k}=D_{\alpha}^{2 \overline{1}} U_{-k}=0, \\
& \overline{\mathbb{D}}_{\alpha} U_{l}=-D_{\alpha}^{1 \overline{2}} U_{l-1}, \quad D_{\alpha}^{2 \overline{1}} U_{l}=\mathbb{D}_{\alpha} U_{l-1}, \quad(-k+1 \leq l \leq k), \\
& D_{\alpha}^{1 \overline{2}} U_{k}=\mathbb{D}_{\alpha} U_{k}=0 .
\end{aligned}
$$

The (ant)arctic multiplets and tropical multiplets are defined by taking $k \rightarrow \infty$ in the complex $\mathcal{O}(k)$ and the real $\mathcal{O}(-k, k)$ multiplets, respectively.

We now consider the Chern-Simons action in $\mathcal{N}=4$ projective superspace. Following the discussion in the previous section, we consider the weight-2 Lagrangian in the left part,

$$
\mathcal{L}_{L}^{(2)}=\frac{k}{8 \pi} \mathcal{V}_{L}^{(0)}\left(z, v_{L}\right) G_{L}^{(2)}\left(z, v_{L}\right)
$$

where $\mathcal{V}_{L}^{(0)}$ is the weight-0 tropical multiplet while $G_{L}^{(2)}$ is the gauge invariant weight-2 real $\mathcal{O}(-1,1)$ multiplet. The constraints for the component superfields in $G_{L}^{(2)}$ are obtained from (5.16) as

$$
\begin{aligned}
& \overline{\mathbb{D}}_{\alpha} \Phi_{L}=0, \\
& \mathbb{D}^{2} L_{L}=\overline{\mathbb{D}}^{2} L_{L}=0, \\
& \mathbb{D}_{\alpha} \bar{\Phi}_{L}=0,
\end{aligned}
$$

where they are projected on the $\mathcal{N}=2$ superspace. Now we represent the gauge invariant multiplet $G_{L}^{(2)}$ by the gauge potential. The closed form of the solution to the $\mathcal{N}=4$ projective superspace constraint was obtained in [23]. The result is

$$
G_{L}^{(2)}\left(v_{L}\right)=-\frac{i}{4} D^{(2) \bar{i} \bar{j}} \oint_{\gamma} \frac{\left(v_{R}, d v_{R}\right)}{2 \pi} \frac{u_{\bar{i}} u_{\bar{j}}}{\left(v_{R}, u_{R}\right)^{2}} \mathcal{V}_{R}^{(0)}\left(v_{R}\right)
$$


or equivalently

$$
G_{L}^{i j}=-\frac{i}{4} \oint_{\gamma} \frac{\left(v_{R}, d v_{R}\right)}{2 \pi} D^{(-2) i j} \mathcal{V}_{R}^{(0)}\left(v_{R}\right)
$$

Here the left $\mathcal{O}(-1,1)$ multiplet is expanded as $G_{L}^{(2)}=G_{L}^{i j} v_{i} v_{j}$. We note that the left multiplet $G_{L}$ is represented by the right multiplet $\mathcal{V}_{R}$ in the $\mathcal{N}=4$ case. Performing the contour integration, we find

$$
G_{L}^{[2]}=\frac{i}{\zeta_{L}}\left(-\frac{1}{4} \overline{\mathbb{D}}^{\alpha} \overline{\mathbb{D}}_{\alpha} V_{R, 1}\right)+\frac{i}{2} \overline{\mathbb{D}}^{\alpha} \mathbb{D}_{\alpha} V_{R, 0}+i \zeta_{L}\left(\frac{1}{4} \mathbb{D}^{\alpha} \mathbb{D}_{\alpha} V_{R,-1}\right)
$$

where we have defined $G_{L}^{(2)}=\left(v^{1}\right)^{2}\left(i \zeta_{L}\right) G_{L}^{[2]}$ and the component fields of $\mathcal{V}_{R}^{[0]}$ in the LindströmRoček gauge:

$$
\mathcal{V}_{R}^{[0]}=\frac{1}{\zeta_{R}} V_{R,-1}+V_{R, 0}+\zeta_{R} V_{R, 1}
$$

The detailed calculations are found in Appendix B. The same analysis is applied to the right multiplet $G_{R}^{(2)}$. Therefore the $\mathcal{N}=4$ Chern-Simons action in the $\mathcal{N}=2$ superspace is given by

$$
\begin{aligned}
S_{C S}= & \frac{i k}{16 \pi} \int d^{3} x d^{4} \theta V_{L, 0} \overline{\mathbb{D}}^{\alpha} \mathbb{D}_{\alpha} V_{R, 0}+\frac{i k}{8 \pi} \int d^{3} x d^{2} \theta \Phi_{L} \Phi_{R}+\frac{i k}{8 \pi} \int d^{3} x d^{2} \bar{\theta} \bar{\Phi}_{L} \bar{\Phi}_{R} \\
& +(L \leftrightarrow R) .
\end{aligned}
$$

The calculation is the same with the $\mathcal{N}=3$ case.

We note that there are two gauge potentials associated with the left and right parts. From the construction, we find that each part is invariant under the two independent $U(1)$ gauge transformations. Therefore the gauge group of the $\mathcal{N}=4$ superconformal Chern-Simons action is interpreted as $U(1) \times U(1)$. The gauge coupling constants for these gauge groups should be the same due to the $S O(4)_{R}$ invariance. This situation is different from the $\mathcal{N}=3$ case where we can construct the gauge invariant action with a single $U(1)$ gauge group. We need more than one gauge potential to construct $\mathcal{N} \geq 4$ supersymmetric Chern-Simons theories. There is a natural interpretation for this gauge group enhancement. It has been discussed that the $\mathcal{N} \geq 4$ supersymmetry completion of a pure Chern-Simons term is impossible provided that there is only one gauge potential. One needs multiple gauge potentials to make the action be invariant under $\mathcal{N} \geq 4$ supersymmetries [2, 3, 4]. We therefore have to introduce more than single gauge group or may need 3-algebra structure which enable the gauge potential to have multiple components (with 3-algebra indices) [7, 8, 9, 27].

For the matter part, we can construct the $\mathcal{N}=4$ action which includes $U(1)$ gauge interactions. The matter multiplets are introduced as weight-1 left (and right) (ant)arctic multiplets $\Upsilon^{[1]}$. Following the Chern-Simons case, since the left and right parts are almost the same, we focus on the left part. We define the gauge covariant derivative as

$$
\mathcal{D}_{\alpha}^{[1] \bar{k}}=e^{-q \mathcal{V}_{-}} D_{\alpha}^{[1] \bar{k}} e^{q \mathcal{V}_{-}}=e^{q \mathcal{V}_{+}} D_{\alpha}^{[1] \bar{k}} e^{-q \mathcal{V}_{+}},
$$

where we have decomposed the left $U(1)$ vector multiplet $\mathcal{V}_{L}^{[0]}=\mathcal{V}_{+}+\mathcal{V}_{-}$in the same way as the $\mathcal{N}=3$ case. We can show that the matter multiplets $\tilde{\Upsilon}^{[1]}=e^{\mathcal{V}_{+}} \Upsilon^{[1]}$ and $\tilde{\Upsilon}^{[1]}=e^{\mathcal{V}_{-}} \bar{\Upsilon}^{[1]}$ satisfy the gauge covariantized projective constraints:

$$
\mathcal{D}_{\alpha}^{[1] \bar{k}} \tilde{\Upsilon}^{[1]}=\mathcal{D}_{\alpha}^{[1] \bar{k}} \tilde{\Upsilon}^{[1]}=0
$$


Using the anticommutation relations of the gauge covariant derivatives in Appendix $\mathrm{C}$ and the constraints (5.25), one can construct the matter action together with the right part as in the same procedure in $\mathcal{N}=3$ case.

Finally we comment on the classification of the multiplets and superconformal invariant action in the $\mathcal{N}=4$ projective superspace. In this paper, we consider the left and right projective multiplets. They can be defined independently and the superconformal invariant action (5.12) is just the sum of the left and right parts. However, one can consider multiplets constructed by the products of the left and right multiplets, called hybrid projective multiplets with weight $(n, m)$ :

$$
Q^{(n, m)}=Q_{L}^{(n)}\left(v_{L}\right) Q_{R}^{(m)}\left(v_{R}\right),
$$

where $Q_{L}^{(n)}\left(Q_{R}^{(m)}\right)$ is a left (right) projective multiplet with weight $n(m)$. The hybrid projective multiplets satisfy the analyticity condition determined by the new covariant derivative $D_{\alpha}^{(1,1)}=$ $v_{i} v_{\bar{k}} D_{\alpha}^{i \bar{k}}$ :

$$
D_{\alpha}^{(1,1)} Q^{(n, m)}=0
$$

The $\mathcal{N}=4$ superconformal invariant action constructed by the hybrid projective multiplets is discussed in [23]. It would be interesting to study $\mathcal{N} \geq 4$ Chern-Simons-matter models by using the hybrid projective multiplets. We leave this possibility to future works.

\section{Conclusion and discussions}

We have constructed the $\mathcal{N}=3$ superconformal Chern-Simons matter theories with Abelian gauge group in the three-dimensional projective superspace. The weight-2 Lagrangian is given by the product of the weight-0 tropical multiplet $\mathcal{V}^{(0)}$ and the gauge invariant weight-2 $\mathcal{O}(-1,1)$ multiplet $G^{(2)}$. We have solved the constraints for the $\mathcal{O}(-1,1)$ multiplet and expressed its component superfields by those of the tropical multiplet. We have also constructed the matter action interacting with the gauge fields. The gauge covariant derivatives are defined by using the tropical multiplet and the matter (ant)arctic multiplets satisfy the gauge covariantized projective constraints.

These constructions of the actions are generalized to the $\mathcal{N}=4$ cases. In order to introduce the R-symmetry group $S O(4)_{R} \sim S U(2) \times S U(2) / \mathbb{Z}_{2}$, we have introduced the mirror $\mathbb{C} P^{1} \mathrm{~S}$ and considered the $\mathcal{N}=4$ superconformal projective superspace. The Chern-Simons and the matter actions are constructed as in the similar way for the $\mathcal{N}=3$ cases. We need to introduce two vector potentials for the $\mathcal{N}=4$ superconformal invariant action. This fact leads to the result that the gauge group of the theory is actually the product of the two groups, namely, $U(1) \times U(1)$.

A few comments on the non-Abelian generalizations of our constructions are in order. Let us try to find the $\mathcal{N}=3$ superconformal Chern-Simons-matter action with non-Abelian gauge groups in the projective superspace. A natural way for the non-Abelian generalization of the $\mathcal{N}=3$ Chern-Simons action (4.1) is to replace the projective multiplets $\mathcal{V}^{(0)}$ and $G^{(2)}$ by the ones with adjoint representations of a non-Abelian group. The Lagrangian may be given by

$$
\mathcal{L}^{(2)}=\frac{k}{8 \pi} \operatorname{Tr}\left[\mathcal{V}^{(0)}(z, v) G^{(2)}(z, v)\right]
$$


where the trace is taken over the adjoint representation of the gauge group. As in the Abelian case, one may try to find solutions to the projective superspace constraints $D_{\alpha}^{(2)} G^{(2)}=0$ and express the $\mathcal{N}=2$ components in $G^{(2)}$ in terms of those in $\mathcal{V}^{(0)}$. Using this solution, the Lagrangian (6.1) should reproduce the $\mathcal{N}=3$ Chern-Simons action (2.5) in $\mathcal{N}=2$ superspace. However, we find that the straightforward generalization of the Abelian solution (4.10) to the non-Abelian case does not work. Even more, the complicated dependence on the auxiliary variable $t$ in (2.5) should be incorporated with the action in the projective superspaces.

The formulation of non-Abelian Chern-Simons theories in the $\mathcal{N}=4$ projective superspace is also an interesting problem. The $\mathcal{N} \geq 4$ superconformal Chern-Simons theories with bifundamental matters have been studied intensively in the context of the world-volume effective theories of M2-branes. In order to construct the $\mathcal{N}=4$ superconformal action for the matter fields with the bi-fundamental representation, one may need to introduce the hybrid projective multiplets. This is because the bi-fundamental matters should couple two gauge potentials simultaneously. We explore these possibilities in the future works.

Finally, let us comment on the construction of the $\mathcal{N}=4$ Chern-Simons-matter model discussed in 24]. In 24, manifestly $\mathcal{N}=4$ supersymmetric construction of the BaggerLambert-Gustavsson type action was studied. The action is based on the 3-algebra gauge invariance and is formulated in the $\mathcal{N}=4$ projective superspace. This $\mathcal{N}=4$ projective superspace is defined by the dimensional reduction of the one in four dimensions and $S O(4)_{R} \sim$ $S U(2) \times S U(2) / \mathbb{Z}_{2}$ R-symmetry is not manifest. There are only one matter multiplet and one vector multiplet in the action and the mirror pairs (left or right multiplets) do not exist, which are necessary to keep $\mathcal{N}=4$ superconformal invariance. Consequently, it is unclear whether their action is $\mathcal{N}=4$ superconformal invariant or not. It would be interesting to formulate $\mathcal{N} \geq 4$ Chern-Simons-matter theories based on the 3 -algebra gauge groups. Our construction may be applicable to the 3 -algebra gauge groups. We hope we come back to this problem in the future researches.

\section{Acknowledgments}

The hospitality of the University of Western Australia during the first stage of this project is gratefully acknowledged. M.A. thanks S.M. Kuzenko for discussion and giving useful comments. The authors would like to thank Y. Imamura for giving us a comment on pure supersymmetric Chern-Simons theories. The work of M. A. is supported in part by the Research Program MSM6840770029 and by the project of International Cooperation ATLAS-CERN of the Ministry of Education, Youth and Sports of the Czech Republic. The work of S. S. is supported by the Research Fellowship of the Japan Society for the Promotion of Science (JSPS).

\section{A Conventions and notations in three-dimensional su- perspaces}

This appendix provides the basic conventions and notations of ordinary superspaces in three dimensions. We use the convention of the three-dimensional metric $\eta_{m n}=\operatorname{diag}(-1,+1,+1)$ with $m, n$ run from 0 to 2 . The three-dimensional $\mathcal{N}=2$ superspace is represented by the coordinate $z^{A}=\left(x^{m}, \theta^{\alpha}, \bar{\theta}^{\alpha}\right)$ where $\theta, \bar{\theta}$ are two component spinors. The gamma matrices 
are defined by $\left(\gamma^{m}\right)_{\alpha}{ }^{\beta}=\left(i \tau^{2}, \tau^{1}, \tau^{3}\right)$ which satisfies $\left\{\gamma^{m}, \gamma^{n}\right\}=2 \eta^{m n} . \tau^{I}(I=1,2,3)$ are the Pauli matrices. The spinor indices are raised and lowered by the anti-symmetric symbol $\varepsilon^{12}=-\varepsilon_{12}=1$. The supercovariant derivatives in $\mathcal{N}=2$ superspace are defined by

$$
\mathbb{D}_{\alpha}=\partial_{\alpha}+i\left(\gamma^{m} \bar{\theta}\right)_{\alpha} \partial_{m}, \quad \overline{\mathbb{D}}_{\alpha}=-\bar{\partial}_{\alpha}-i\left(\theta \gamma^{m}\right)_{\alpha} \partial_{m}
$$

These satisfy the following relations

$$
\left\{\mathbb{D}_{\alpha}, \overline{\mathbb{D}}_{\beta}\right\}=-2 i \gamma_{\alpha \beta}^{m} \partial_{m}, \quad\left\{\mathbb{D}_{\alpha}, \mathbb{D}_{\beta}\right\}=\left\{\overline{\mathbb{D}}_{\alpha}, \overline{\mathbb{D}}_{\beta}\right\}=0 .
$$

The Grassmann measure of integration is defined by

$$
d^{2} \theta=-\frac{1}{4} d \theta^{\alpha} d \theta_{\alpha}, \quad d^{2} \bar{\theta}=-\frac{1}{4} d \bar{\theta}^{\alpha} d \bar{\theta}_{\alpha}, \quad d^{4} \theta=d^{2} \theta d^{2} \bar{\theta},
$$

such that they satisfy

$$
\int d^{2} \theta \theta^{2}=1, \quad \int d^{2} \bar{\theta} \bar{\theta}^{2}=1, \quad \int d^{4} \theta \theta^{2} \bar{\theta}^{2}=1 .
$$

Within the space-time integration, the following relation holds,

$$
\int d^{4} \theta F(z)=\left.\frac{1}{16}\left(\mathbb{D}^{2} \overline{\mathbb{D}}^{2} F(z)\right)\right|_{\theta=\bar{\theta}=0}
$$

where $F(z)$ is a superfield. The chiral and anti-chiral coordinates are defined by

$$
x_{L}^{m}=x^{m}+i \theta \gamma^{m} \bar{\theta}, \quad x_{R}^{m}=x^{m}-i \theta \gamma^{m} \bar{\theta} .
$$

We use the following relations among $\mathcal{N}=2, \mathcal{N}=3$ and $\mathcal{N}=4$ superspaces [22]:

$$
\begin{aligned}
& \theta^{\alpha}=\theta_{11}^{\alpha}=\theta_{1 \overline{1}}^{\alpha}, \quad \bar{\theta}^{\alpha}=\theta_{22}^{\alpha}=\theta_{2 \overline{2}}^{\alpha}, \\
& \mathbb{D}_{\alpha}=D_{\alpha}^{11}=D_{\alpha}^{1 \overline{1}}, \quad \overline{\mathbb{D}}_{\alpha}=-D_{\alpha}^{22}=-D_{\alpha}^{2 \overline{2}} .
\end{aligned}
$$

The $\mathcal{N}=2$ projection of $\mathcal{N}=3,4$ superfields $\Phi\left(z^{A}\right)$ is defined by

$$
\Phi\left|=\Phi\left(z^{A}\right)\right|_{\theta_{\perp}=0},
$$

where $\theta_{\perp}$ is $\theta_{12}(\mathcal{N}=3)$ or $\theta_{1 \overline{2}}, \theta_{2 \overline{1}}(\mathcal{N}=4)$.

\section{B Solutions to the constraint}

In this section, the detailed calculations of the integral in (4.11) and its $\mathcal{N}=4$ counterpart (5.20) are shown.

We fix $u_{i}=(1,0)$. The integral measure is $v^{i} d v_{i}=-\left(v^{1}\right)^{2} d \zeta$ and the products of the supercovariant derivatives are

$$
\begin{aligned}
& D^{(-2) \alpha} D_{\alpha}^{(-2)}=\frac{1}{\left(v^{1}\right)^{2}}\left(-\zeta \mathbb{D}^{2}+\mathbb{D} D^{12}\right), \\
& D^{(-2) \alpha} D_{\alpha}^{(0)}=\frac{1}{\left(v^{1}\right)^{2}}\left(-\frac{1}{2} \zeta \mathbb{D}^{2}-\frac{1}{2 \zeta} \mathbb{D} \overline{\mathbb{D}}\right), \\
& D^{(0) \alpha} D_{\alpha}^{(0)}=\zeta^{2} \mathbb{D}^{2}-2 \zeta \mathbb{D} D^{12}+\left(D^{12}\right)^{2} .
\end{aligned}
$$


One can eliminate $D^{12}$ in each expression using the relation $D^{12}=-\frac{1}{2 \zeta}\left(D^{[2]}+\overline{\mathbb{D}}-\zeta^{2} \mathbb{D}\right)$. We start from the first term in (4.11):

$$
\begin{aligned}
& -\frac{1}{8 \pi i} \oint_{\gamma}(v, d v)\left(-\frac{i}{2}\right)(w, v)^{2}\left(D^{(-2)}\right)^{2} \mathcal{V}^{(0)}(v) \\
= & -\frac{i}{2} \frac{1}{8 \pi i} \oint_{\gamma} d \zeta_{v}\left(w_{1}^{2}+2 \zeta_{v} w_{1} w_{2}+\zeta_{v}^{2} w_{2}^{2}\right) \mathbb{D}^{2}\left(\frac{1}{\zeta_{v}} V_{-1}+V_{0}+\zeta_{v} V_{1}\right) \\
= & -\frac{i}{8} w_{1}^{2} \mathbb{D}^{2} V_{-1},
\end{aligned}
$$

where we have used the projective superspace constraint $D^{[2]} \mathcal{V}^{[0]}=0$ and defined $\zeta_{v}=\frac{v^{2}}{v^{1}}$. The second term in (4.11) is

$$
\begin{aligned}
& -\frac{1}{8 \pi i} \oint_{\gamma}(v, d v) 8 \frac{(w, v)(w, u)}{(v, u)} D^{(-2)} D^{(0)} \mathcal{V}^{(0)} \\
= & \frac{1}{\pi i} \oint_{\gamma} d \zeta_{v}\left(w_{1} w_{2}+\zeta_{v} w_{2}^{2}\right)\left(-\frac{1}{2} \zeta_{v} \mathbb{D}^{2}-\frac{1}{2 \zeta_{v}} \mathbb{D} \overline{\mathbb{D}}\right)\left(\frac{1}{\zeta_{v}} V_{-1}+V_{0}+\zeta_{v} V_{1}\right) \\
= & w_{1} w_{2} \mathbb{D} \overline{\mathbb{D}} V_{0} .
\end{aligned}
$$

The third term is

$$
\begin{aligned}
& -\frac{1}{8 \pi i} \oint_{\gamma}(v, d v) 2 i \frac{(w, u)^{2}}{(v, u)^{2}}\left(D^{(0)}\right)^{2} \mathcal{V}^{(0)} \\
= & \frac{1}{4 \pi} \oint_{\gamma} d \zeta_{v}\left(w_{2}\right)^{2} \frac{1}{4 \zeta_{v}^{2}} \overline{\mathbb{D}}^{2}\left(\frac{1}{\zeta_{v}} V_{-1}+V_{0}+\zeta_{v} V_{1}\right) \\
= & \frac{i}{2}\left(w_{2}\right)^{2} \overline{\mathbb{D}}^{2} V_{1} .
\end{aligned}
$$

From these results we find

$$
G^{[2]}(w)=\frac{i}{\zeta_{w}}\left(\frac{i}{8} \overline{\mathbb{D}}^{2} V_{1}\right)+i \overline{\mathbb{D}} \mathbb{D} V_{0}+i \zeta_{w}\left(-\frac{i}{8} \mathbb{D}^{2} V_{-1}\right)
$$

Therefore (4.10) is obtained.

Next, we calculate the integral (5.20). The left $\mathcal{O}(-1,1)$ multiplet $G_{L}^{(2)}=G^{i j} v_{i} v_{j}$ is expanded as

$$
\begin{aligned}
G_{L}^{(2)} & =\left(v^{1}\right)^{2}\left(i \zeta_{L}\right)\left(-i \zeta_{L} G^{11}+2 i G^{12}-i \zeta_{L}^{-1} G^{22}\right) \\
& \equiv\left(v^{1}\right)^{2}\left(i \zeta_{L}\right) G_{L}^{[2]}
\end{aligned}
$$

Then, we find

$$
\begin{aligned}
G^{11} & =\frac{1}{4} \oint_{\gamma} \frac{\left(v^{\overline{1}}\right)^{2} d \zeta_{R}}{2 \pi i} \frac{1}{\left(v^{\overline{1}}\right)^{2}}\left(D^{1 \overline{1}}\right)^{2}\left(\frac{1}{\zeta_{R}} V_{-1}^{R}+V_{0}^{R}+\zeta_{R} V_{1}^{R}\right) \\
& =\frac{1}{4} \mathbb{D}^{2} V_{-1}^{R}
\end{aligned}
$$


Similarly, the other components are

$$
\begin{aligned}
G^{12} & =\frac{1}{4} \oint_{\gamma} \frac{d \zeta_{R}}{2 \pi i} D^{1 \overline{1}} D^{1 \overline{2}}\left(\frac{1}{\zeta_{R}} V_{-1}^{R}+V_{0}^{R}+\zeta_{R} V_{1}^{R}\right) \\
& =-\frac{1}{4} \overline{\mathbb{D}} \mathbb{D} V_{0}^{R}, \\
G^{22} & =\frac{1}{4} \oint_{\gamma} \frac{d \zeta_{R}}{2 \pi i}\left(D^{1 \overline{2}}\right)^{2}\left(\frac{1}{\zeta_{R}} V_{-1}^{R}+V_{0}^{R}+\zeta_{R} V_{1}^{R}\right) \\
& =\frac{1}{4} \mathbb{D}^{2} V_{1}^{R},
\end{aligned}
$$

where we have used the $\mathcal{N}=4$ projective superspace constraints on $\mathcal{V}_{R}^{(0)}$. Finally we obtain (5.21).

\section{Anticommutation relations of supercovariant deriva- tives}

The $\mathcal{N}=3$ super gauge covariant derivatives satisfy the following anticommutation relations

$$
\begin{aligned}
\left\{\mathcal{D}_{\alpha}, \mathcal{D}_{\beta}\right\} & =0 \\
\left\{\overline{\mathcal{D}}_{\alpha}, \overline{\mathcal{D}}_{\beta}\right\} & =2 \overline{\mathbb{D}}_{(\alpha} D_{\beta)}^{12} V_{-1} \\
\left\{\mathcal{D}_{\alpha}^{12}, \mathcal{D}_{\beta}^{12}\right\} & =\left\{D_{\alpha}^{12}, D_{\beta}^{12}\right\}+\frac{1}{2}\left\{D_{\alpha}^{12}, D_{\beta}^{12}\right\} V_{0}-\frac{1}{2} D_{(\alpha}^{12} \mathbb{D}_{\beta)} V_{-1}, \\
\left\{\mathcal{D}_{\alpha}, \mathcal{D}_{\beta}^{12}\right\} & =-\frac{1}{2} \mathbb{D}_{\alpha} \mathbb{D}_{\beta} V_{-1} \\
\left\{\mathcal{D}_{\alpha}, \overline{\mathcal{D}}_{\beta}\right\} & =\left\{\mathbb{D}_{\alpha}, \overline{\mathbb{D}}_{\beta}\right\}+\frac{1}{2}\left\{\mathbb{D}_{\alpha}, \overline{\mathbb{D}}_{\beta}\right\} V_{0}+2 \mathbb{D}_{\alpha} D_{\beta}^{12} V_{-1}, \\
\left\{\overline{\mathcal{D}}_{\alpha}, \mathcal{D}_{\beta}^{12}\right\} & =-\frac{1}{2} \overline{\mathbb{D}}_{\alpha} \mathbb{D}_{\beta} V_{-1}+2 D_{\beta}^{12} D_{\alpha}^{12} V_{-1},
\end{aligned}
$$

where we have taken $q=1$ for simplicity.

For the $\mathcal{N}=4$ case, the gauge covariant derivative in the left sector is expanded as

$$
\mathcal{D}_{\alpha}^{[1] \bar{k}}=\mathcal{D}_{\alpha}^{2 \bar{k}}-\zeta \mathcal{D}_{\alpha}^{1 \bar{k}}
$$

where we have defined

$$
\begin{aligned}
\mathcal{D}_{\alpha}^{2 \bar{k}} & =D_{\alpha}^{2 \bar{k}}+\frac{q}{2} D_{\alpha}^{2 \bar{k}} V_{0}-q D_{\alpha}^{1 \bar{k}} V_{-1}, \\
\mathcal{D}_{\alpha}^{1 \bar{k}} & =D_{\alpha}^{1 \bar{k}}+\frac{q}{2} D_{\alpha}^{1 \bar{k}} V_{0} .
\end{aligned}
$$


These satisfy the following anticommutation relations with $q=1$ :

$$
\begin{aligned}
& \left\{\mathcal{D}_{\alpha}^{1 \overline{1}}, \mathcal{D}_{\beta}^{1 \overline{1}}\right\}=0 \\
& \left\{\mathcal{D}_{\alpha}^{1 \overline{1}}, \mathcal{D}_{\beta}^{1 \overline{2}}\right\}=0 \\
& \left\{\mathcal{D}_{\alpha}^{1 \overline{1}}, \mathcal{D}_{\beta}^{2 \overline{1}}\right\}=-\mathbb{D}_{\alpha} \mathbb{D}_{\beta} V_{-1} \\
& \left\{\mathcal{D}_{\alpha}^{1 \overline{1}}, \mathcal{D}_{\beta}^{2 \overline{2}}\right\}=-\left\{\mathbb{D}_{\alpha}, \overline{\mathbb{D}}_{\beta}\right\}-\frac{1}{2}\left\{\mathbb{D}_{\alpha}, \overline{\mathbb{D}}_{\beta}\right\} V_{0}-\mathbb{D}_{\alpha} D_{\beta}^{1 \overline{2}} V_{-1}, \\
& \left\{\mathcal{D}_{\alpha}^{1 \overline{2}}, \mathcal{D}_{\beta}^{1 \overline{2}}\right\}=0 \\
& \left\{\mathcal{D}_{\alpha}^{1 \overline{2}}, \mathcal{D}_{\beta}^{2 \overline{1}}\right\}=\left\{D_{\alpha}^{1 \overline{2}}, D_{\beta}^{2 \overline{1}}\right\}+\frac{1}{2}\left\{D_{\alpha}^{1 \overline{2}}, D_{\beta}^{2 \overline{1}}\right\} V_{0}-D_{\alpha}^{1 \overline{2}} \mathbb{D}_{\beta} V_{-1}, \\
& \left\{\mathcal{D}_{\alpha}^{1 \overline{2}}, \mathcal{D}_{\beta}^{2 \overline{2}}\right\}=-D_{\alpha}^{1 \overline{2}} D_{\beta}^{1 \overline{2}} V_{-1} \\
& \left\{\mathcal{D}_{\alpha}^{2 \overline{1}}, \mathcal{D}_{\beta}^{2 \overline{1}}\right\}=-D_{(\alpha}^{2 \overline{1}} \mathbb{D}_{\beta)} V_{-1}, \\
& \left\{\mathcal{D}_{\alpha}^{2 \overline{1}}, \mathcal{D}_{\beta}^{2 \overline{2}}\right\}=-D_{\alpha}^{2 \overline{1}} D_{\beta}^{1 \overline{2}} V_{-1}+\overline{\mathbb{D}}_{\beta} \mathbb{D}_{\alpha} V_{-1}, \\
& \left\{\mathcal{D}_{\alpha}^{2 \overline{2}}, \mathcal{D}_{\beta}^{2 \overline{2}}\right\}=\overline{\mathbb{D}}_{(\alpha} D_{\beta)}^{1 \overline{2}} V_{-1} .
\end{aligned}
$$

Similar definition of the gauge covariant derivative is applied to the right sector.

\section{References}

[1] H. -C. Kao, K. -M. Lee, Phys. Rev. D46 (1992) 4691 hep-th/9205115.

[2] H. Nishino, S. J. Gates, Jr., Int. J. Mod. Phys. A8 (1993) 3371.

[3] H. Nishino and S. J. J. Gates, Nucl. Phys. B 480 (1996) 573 arXiv:hep-th/9606090.

[4] R. Brooks and S. J. J. Gates, Nucl. Phys. B 432 (1994) 205 arXiv:hep-th/9407147.

[5] J. Bagger and N. Lambert, Phys. Rev. D 75 (2007) 045020 hep-th/0611108,

Phys. Rev. D 77 (2008) 065008 [arXiv:0711.0955 [hep-th]],

JHEP 0802 (2008) 105 [arXiv:0712.3738 [hep-th]].

[6] A. Gustavsson, Nucl. Phys. B 811 (2009) 66 [arXiv:0709.1260 [hep-th]].

[7] M. Van Raamsdonk, JHEP 0805 (2008) 105 arXiv:0803.3803 [hep-th]].

[8] K. Hosomichi, K. -M. Lee, S. Lee, S. Lee, J. Park, JHEP 0807 (2008) 091 arXiv:0805.3662 [hep-th]], JHEP 0809 (2008) 002 [arXiv:0806.4977 [hep-th]].

[9] O. Aharony, O. Bergman, D. L. Jafferis and J. Maldacena, JHEP 0810 (2008) 091 arXiv:0806.1218 [hep-th]].

[10] E. A. Ivanov, Phys. Lett. B 268 (1991) 203.

[11] S. J. J. Gates and H. Nishino, Phys. Lett. B 281 (1992) 72. 
[12] M. Cederwall, JHEP 0809 (2008) 116 [arXiv:0808.3242 [hep-th]], JHEP 0810 (2008) 070 [arXiv:0809.0318 [hep-th]].

[13] A. Galperin, E. Ivanov, V. Ogievetsky, E. Sokatchev, JETP Lett. 40 (1984) 912-916.

[14] A. Galperin, E. Ivanov, S. Kalitsyn, V. Ogievetsky, E. Sokatchev, Class. Quant. Grav. 1 (1984) 469-498.

[15] P. S. Howe, M. I. Leeming, Class. Quant. Grav. 11 (1994) 2843-2852 hep-th/9408062.

[16] B. M. Zupnik, Phys. Lett. B660 (2008) 254-259. [arXiv:0711.4680 [hep-th]], Theor. Math. Phys. 157 (2008) 1550 [arXiv:0802.0801 [hep-th]].

[17] I. L. Buchbinder, E. A. Ivanov, O. Lechtenfeld, N. G. Pletnev, I. B. Samsonov, B. M. Zupnik, JHEP 0903 (2009) 096 [arXiv:0811.4774 [hep-th]].

[18] A. Karlhede, Lindström and M. Roček, Phys. Lett. B147 (1984) 297.

[19] U. Lindström, M. Roček, Commun. Math. Phys. 115 (1988) 21.

[20] U. Lindström, M. Roček, Commun. Math. Phys. 128 (1990) 191.

[21] S. M. Kuzenko, Int. J. Mod. Phys. A 14 (1999) 1737 [hep-th/9806147].

[22] S. M. Kuzenko, J. H. Park, G. Tartaglino-Mazzucchelli and R. Unge, JHEP 1101 (2011) 146 [arXiv:1011.5727 [hep-th]].

[23] S. M. Kuzenko, U. Lindström and G. Tartaglino-Mazzucchelli, JHEP 1103 (2011) 120 arXiv:1101.4013 [hep-th]].

[24] S. Cherkis, V. Dotsenko and C. Saemann, Phys. Rev. D 79 (2009) 086002 arXiv:0812.3127 [hep-th]].

[25] J. H. Schwarz, JHEP 0411 (2004) 078 hep-th/0411077].

[26] D. Gaiotto, X. Yin, JHEP 0708 (2007) 056 [arXiv:0704.3740 [hep-th]].

[27] D. Gaiotto and E. Witten, JHEP 1006 (2010) 097 [arXiv:0804.2907 [hep-th]].

[28] S. M. Kuzenko and W. D. I. Linch, JHEP 0602 (2006) 038 hep-th/0507176].

[29] S. M. Kuzenko, Phys. Lett. B 644 (2007) 88 hep-th/0609078.

[30] F. Gonzalez-Rey and R. von Unge, Nucl. Phys. B 516 (1998) 449 hep-th/9711135. 